\title{
PIV INVESTIGATION OF 3-DIMENSIONAL FLOW IN DROPS ACTUATED BY EWOD
}

\author{
H.-W. Lu ${ }^{1}$, F. Bottausci ${ }^{2}$, A. L. Bertozzi ${ }^{3}$, C. D. Meinhart ${ }^{2}$, and C.-J. Kim ${ }^{1}$ \\ ${ }^{1}$ Mechanical and Aerospace Engineering Department, University of California, Los Angeles, CA, USA \\ ${ }^{2}$ Mechanical Engineering Department, University of California, Santa Barbara, CA, USA \\ ${ }^{3}$ Mathematics Department, University of California, Los Angeles, CA, USA
}

\begin{abstract}
We report an investigation of the flow inside droplets actuated by electrowetting-on-dielectric (EWOD) in air using micro particle image velocimetry (microPIV). Using the continuity equation, we reconstruct the $3 \mathrm{D}$ velocity field from the 2D PIV experimental data. We present some fundamental findings and build valuable insights that will help the design of new sophisticated EWOD microfluidic devices for mixing purpose.
\end{abstract}

\section{INTRODUCTION}

Despite the recent interest in the digital microfluidics based on electrowetting-on-dielectric (EWOD) [1, 2]. Fundamental understanding of the fluid dynamics in the droplet remains limited. The available flow simulations, including commercial codes, are all based on a fully developed flow profile in a thin flat droplet $[3,4]$. It is well known that the flow in a thin and flat droplet is, to the leading order, irrotational[5], thus inefficient for mixing. Therefore it is necessary to understand the fluid dynamics in a thick, 3D, droplet. Fowler et al. [6] proposed a simple movement pattern in parallel-plate EWOD devices to mix drops in air. Paik et al. [7] studied the mixing performances of different movement patterns of aqueous drops in parallel-plate EWOD devices filled with silicone oil. We expect the flow pattern in a droplet in air to be different from a droplet in oil, due to the significant viscosity difference of the external fluid. Dyebased flow visualization must be considered with great care, since the fluorescent dye creates a surface tension gradient that induces a surface flow. To obtain a basic understanding of the flow in a 3D droplet actuated by EWOD, we characterize the microfluidic flow inside of a thick droplet using micro-PIV [8,9]. The droplet is uniformly seeded with polystyrene beads, thus free of any surface tension gradient. We imaged the horizontal velocity fields on 7 planes of different heights from the bottom substrate. The 3D velocity field reconstructed from the 2D PIV experimental data presents some fundamental findings that will help designing of new sophisticated EWOD microfluidic devices in the future.

\section{EXPERIMENTAL SETUP}

The parallel-plate EWOD devices are fabricated in the Micro and Nano Manufacturing Laboratory using Nanoelectronics Research Facility (Nanolab) in University of California, Los Angeles (UCLA). Square shaped electrodes, each having $600 \mu \mathrm{m} \times 600 \mu \mathrm{m}$ of area, are patterned on top of indium-tin oxide (ITO) coated glass wafers, which serves as the bottom substrates of the parallel-plate EWOD devices. A $500 \mathrm{~nm}$ blanket layer of silicon oxide is deposited by PECVD. A final coating of $\sim 250$ nm-thick Cytop ${ }^{\circledR}$ fluoropolymer (Asahi Glass, Japan) is deposited by spin coating a $6 \%$ solvated solution on the wafer. The top substrate of the electrowetting device is a cleaved ITO glass wafer. Spin coating a $3 \%$ solvated solution gives a $\sim 50$ nm Cytop ${ }^{\circledR}$ layer on the top substrate.

The EWOD devices are brought to the Microfluidics Laboratory in University of California, Santa Barbara (UCSB). The devices and the electronic control are integrated with the micro-PIV system as illustrated in Fig. 1. The fluid used is deionized water seeded with Nile red colored fluorescent polystyrene microbeads of $2 \mu \mathrm{m}$ in diameter (Invitrogen) at $0.01 \%$ solid concentration. The resulting particle density is $2 \times 10^{7}$ particles $/ \mathrm{mL}$. The user controls the applied potential on each electrode through a digital I/O board (Daqpad-6507, National Instrument) that controls electronic relays that pass the high voltage and the ground potential from the voltage source to the electrodes. The digital I/O board also sends a $5 \mathrm{~V}$ falling edge as the synchronization signal to the computer controlling the micro-PIV setup. The pulses of two frequency-doubled Nd:YAG lasers emitting green light $(\lambda=$ $523 \mathrm{~nm}$ ) are combined, directed through an optical fiber into a Nikon TE200 inverted microscope. The pulses are reflected by a long-pass filter cube onto the EWOD device and excite the fluorescent microbeads. The fluorescent emission is filtered by the long-pass filter cube and captured by a cooled $1030 \times 1300 \times 12$ bits interline transfer CCD camera through a $\mathrm{NA}=0.45,10 \mathrm{X}$ objective lens, resulting in approximately 25 $\mu \mathrm{m}$ of depth of focus.

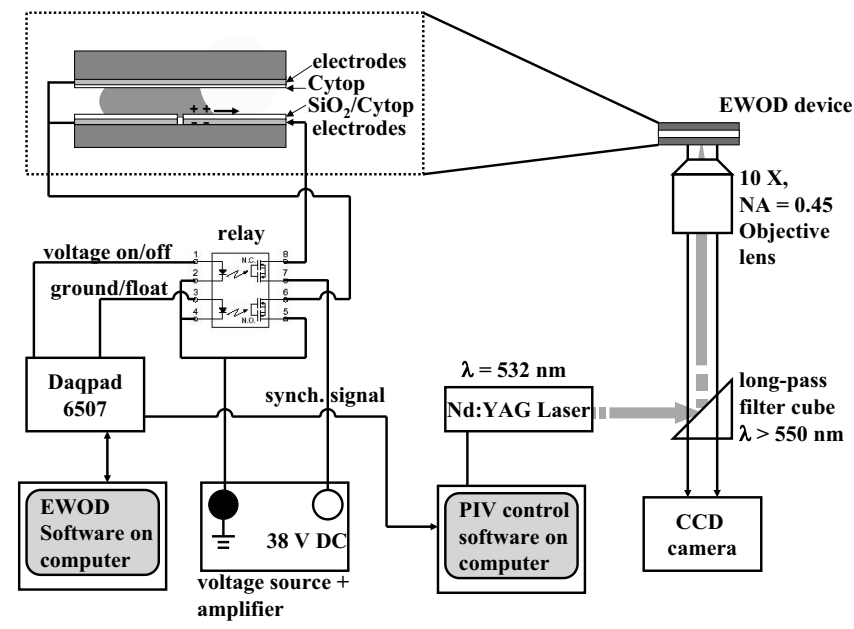

Figure 1: EWOD device and PIV experimental setups. 


\section{PROCEDURE}

An adhesive well made of SecureSeal (Grace BioLab) is used on the bottom substrate to surround the EWOD area and to maintain a substrate separation of 388 $\mu \mathrm{m}$. One droplet of $0.1 \mu \mathrm{l}$ seeded fluid is deposited on the device, along with six $5 \mu$ droplets of deionized water to prevent evaporation. The device is then completed in the pressure contact packaging developed by Fan et al. [10]. The pressure contact packaging presses the top plate onto the SecureSeal well and seals the device with the drops in it. The seeded droplet constrained by the top and the bottom substrates and has a disc diameter of approximately $800 \mu \mathrm{m}$. The experiment repeatedly actuates the droplet back and forth between two electrodes as illustrated in Fig. 2. The droplet initially sits on top of electrode B. At time $t=0$, a 38 volt DC potential is applied to electrode $A$ with electrode $B$ grounded, and a synchronization signal is sent to the micro-PIV setup. At $t=150 \mathrm{~ms}$ after receiving the synchronization signal, the micro-PIV setup fires the lasers and captures two images of the moving droplet with $5-20 \mathrm{~ms}$ of delay between them. At $t=300 \mathrm{~ms}$, the actuation potential is switched onto electrode $\mathrm{B}$ for a duration of $300 \mathrm{~ms}$ with electrode A grounded to move the droplet back to electrode B. Starting from $t=600 \mathrm{~ms}$, both electrodes are grounded for $1400 \mathrm{~ms}$ before the next cycle of movement/image capture is initiated. Sixty sets of image pairs are captured in this manner. Using the micro-PIV software [9], we analyze the image pairs using an interrogation region of 96 pixels $\times 96$ pixels, which yields $2 \mathrm{D}$ velocity data at $32 \mu \mathrm{m} \times 32 \mu \mathrm{m}$ resolution. We repeat the procedure on 7 planes above the bottom substrate, $z=52 \mu \mathrm{m}, 100 \mu \mathrm{m}, 148 \mu \mathrm{m}, 196 \mu \mathrm{m}, 244 \mu \mathrm{m}$, $292 \mu \mathrm{m}$, and $340 \mu \mathrm{m}$, to obtain a stack of $2 \mathrm{D}$ velocity fields.

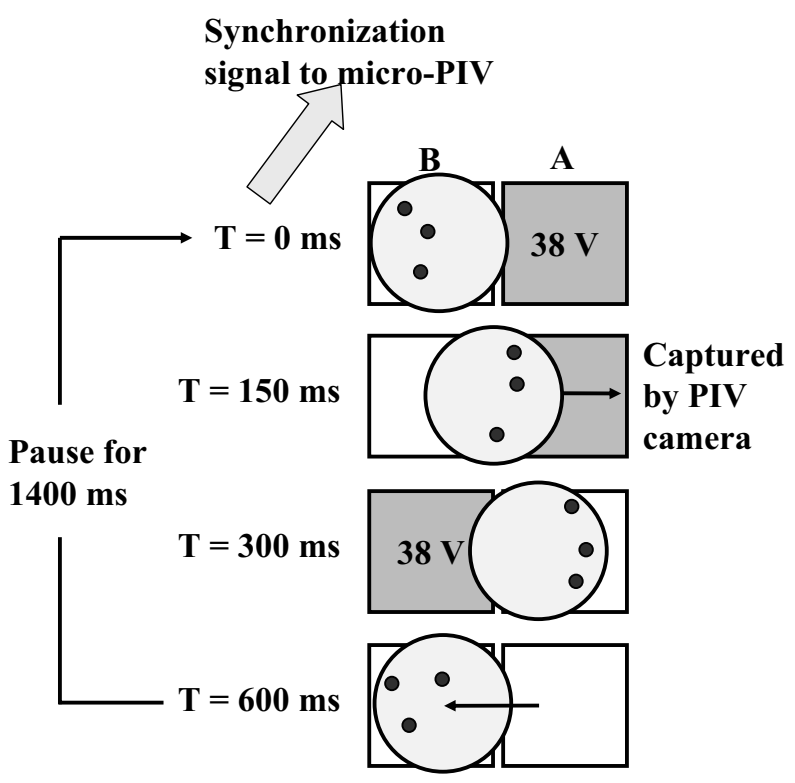

Figure 2: One cycle of actuation sequence of the electrowetting device.

\section{RESULTS}

The droplet moves with a velocity of $\sim 2 \mathrm{~mm} / \mathrm{s}$ in the fixed reference frame. Viewing the $2 \mathrm{D}$ velocity field near the midheight of the drop $(\mathrm{z}=148 \mu \mathrm{m})$, in the reference frame moving with the droplet (Lagrangian frame), clearly shows two internal circulations (Fig. 3) symmetric about the moving axis of the droplet, $\boldsymbol{x}$. The fluid flows forward along the free surface (i.e, the liquid-air interface) of the droplet toward the nose and circulates backward near the center, a flow pattern similar to the one reported by Fowler et al [6].

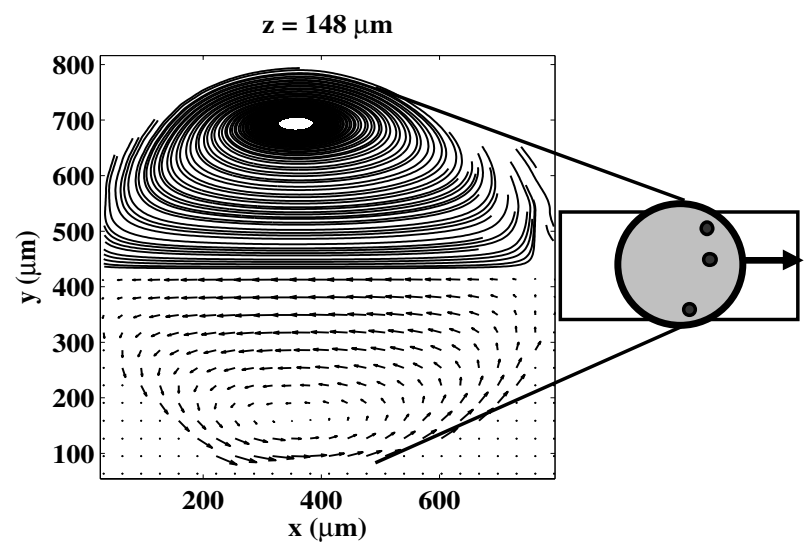

Figure 3: The 2-dimensional velocity field (bottom half) and the streamlines (top half) at height $z=148 \mu \mathrm{m}$.

Using the entire stack of $2 \mathrm{D}$ velocity fields, we obtain the velocity component normal to the substrates, $w$, by first computing the 2D divergence of the horizontal velocity measured on all 7 horizontal planes. Using the continuity equation, the normal gradient of $w$ is

$$
\frac{\partial w}{\partial z}=-\frac{\partial u}{\partial x}-\frac{\partial v}{\partial y}
$$

We interpolate a 7 th order polynomial, $p(z)$, through the data of $\partial w / \partial z$ through all 7 planes,

$$
p(z)=\sum_{n=1}^{7} c_{n} z^{n},
$$

with no-slip boundary condition at $z=388 \mu \mathrm{m}$. Subsequently integrating $p(z)$ gives us an estimate of the normal velocity component. The 3D velocity field is shown in Fig. 4. Indeed, we observe 3D flows in the bulk of the droplet. The flow gains significant normal velocity component as it approaches the drop interface. A strong downward flow due to the electrowetting from the bottom substrate is observed near the nose of the droplet. The fluid near the substrates circulates back into the droplet near the rear of the droplet. The flow is asymmetric across the height of the droplet due to corresponding asymmetric actuation in the current EWOD devices. 
(a)

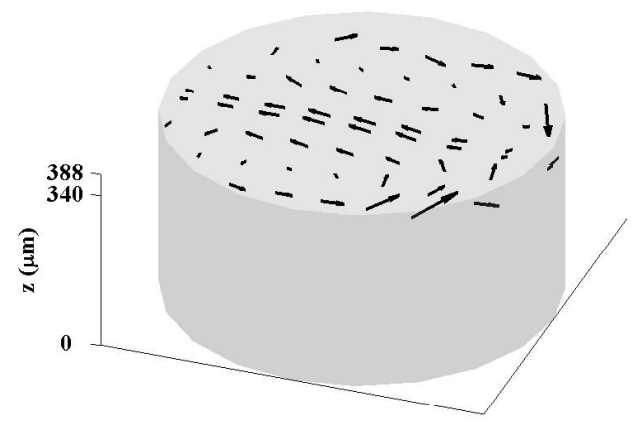

(b)

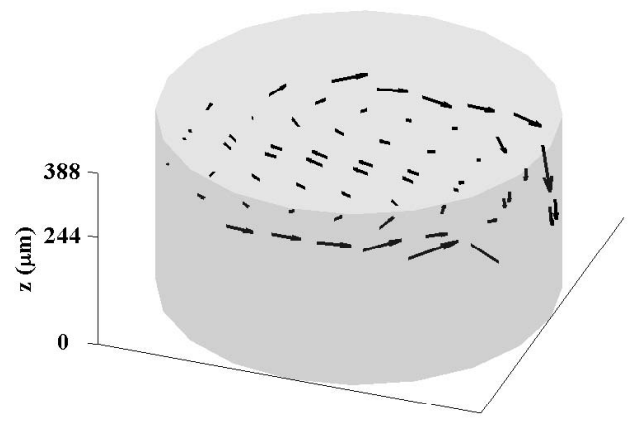

(c)

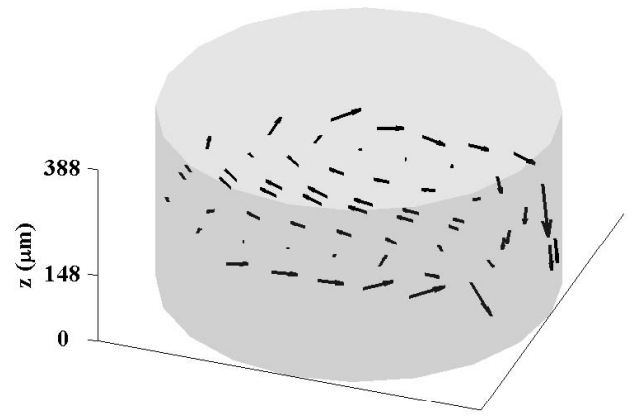

(d)

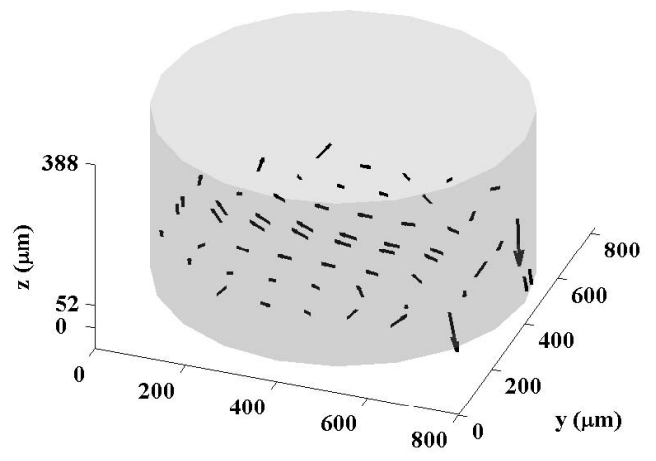

Figure 4: The 3-dimensional velocity field at (a) $z=340$ $\mu \mathrm{m}$; (b) $z=244 \mu \mathrm{m}$; (c) $z=148 \mu \mathrm{m}$; (d) $z=52 \mu \mathrm{m}$.
In addition to the knowledge of the instantaneous velocity field, we need to understand the net effect of the forward-andback droplet motions on the mixing within the droplet. We obtain from the 2D experimental data the overall displacements of the fluid in one cycle of the droplet motion by comparing the positions of the tracer particles between the first images from all 60 image pairs on the horizontal plane at $z=148 \mu \mathrm{m}$. The overall displacement field shown in Fig. 5 is symmetric about the moving axis, $\boldsymbol{x}$, but does not follow the direction of the instantaneous velocity field. The net effect of one actuation cycle is that the fluid enters the horizontal plane near the nose $(x=800 \mu \mathrm{m})$ and the tail $(x=0 \mu \mathrm{m})$ of the droplet. The flow converges toward two points near the front half of the droplet interface and exits out of the horizontal plane. The net displacements of the particles by one actuation cycle are very small $(\sim 10 \mu \mathrm{m})$. This indicates the reverse droplet motion returns the particles near their initial positions, a reversible characteristic of a well-ordered laminar flow. The small displacement may be due to the asymmetry of the actuation cycle, since the droplet waits $1400 \mathrm{~ms}$ on electrode B but not on electrode A. As Fig. 5 shows, transporting the particles over a significant distance $(>100 \mu \mathrm{m})$ requires 15 cycles of droplet actuations. This indicates the forward-andback motion is inefficient for droplet mixing, even if one considers dye with higher diffusion coefficient.

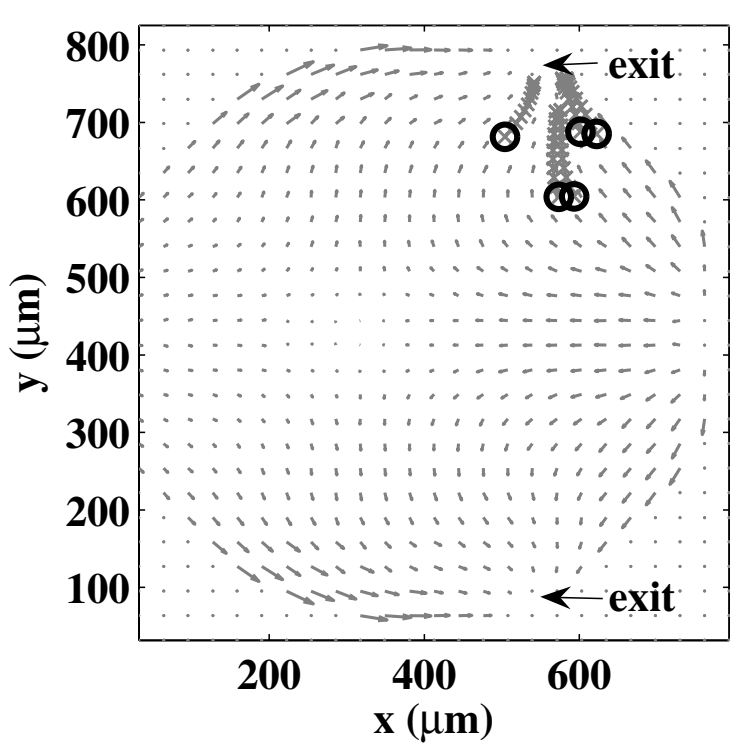

Figure 5: The overall displacements of particles by 15 cycles of the Forward-and-back droplet actuations, starting at random positions denoted by Os. The final position at the end of each cycle is denoted by the $\times$ s.

\section{CONCLUSIONS}

In practice, EWOD device often operate on drops that do not conform to the geometric assumption of a thin, flat 2D drop. As the drop evaporates or cuts, its disc radius to a length scale comparable to the separation height of the device. Our experimental finding shows the flow in a 3D droplet indeed deviates significantly from the parabolic flow profile used in 
current flow simulations. To further our understanding of the fluid dynamics, it is necessary to address the challenges of modeling and simulating a constrained 3D droplet, that couples the no-slip solid boundary condition with the the stress-free interface. Efficient simulations of a 3D drop must also address the challenging issue of growing computational cost that scales on the order of $\mathrm{O}\left(N^{3}\right)$, where $N$ is the number of grid points along one dimension. It would be desirable to develop a reduced dimension model that is computationally tractable while preserving the essential information about the $3 \mathrm{D}$ velocity field. Galerkin methods from numerical analysis may provide an interesting direction to accomplish this. Our 2D data shows two symmetric circulations within the moving droplet. Due to the reversibility of the flow, a simple forward-and-back motion is inefficient for mixing. Effective mixing has to be accomplished through designing irreversible movement patterns to break the symmetry of the circulating flow in the horizontal plane.

\section{ACKNOWLEDGEMENTS}

We thank Professor Pirouz Kavehpour and Mr. Jessie Fowler for valuable discussions on PIV data analysis. This work was funded by ONR grant N000140710431, NSF grant ACI-0321917, NSF grant NIRT CTS-040444, NASA through Institute for Cell Mimetic for Space Exploration (CMISE), and NIH through Pacific Southwest Regional Center of Excellence.

\section{REFERENCES}

[1] J. Lee, H. Moon, J. Fowler, T. Schoellhammer, and C.-J. Kim, "Electrowetting and electrowetting-ondielectric for microscale liquid handling", Sens Actuator A., vol. A95, pp. 259-268, 2002.
[2] M. G. Pollack, R. B. Fair, and A. D. Shenderov, "Electrowetting-based actuation of liquid droplets for microfluidic applications", Appl. Phys. Lett., vol. 77, pp. 1725-1726, 2000.

[3] H.-W. Lu, K. Glasner, A.L. Bertozzi, and C.-J. Kim, "Electrowetting in a Hele-Shaw Cell", J. Fluid Mech., vol. 590, pp. 411-435, 2007.

[4] S.W. Walker, and B. Shapiro, "Modeling the fluid dynamics of electrowetting on dielectric (EWOD)", $J$. Microelectromech. Syst., vol. 15, pp. 986-1000, 2006.

[5] D.J. Acherson, Elementary Fluid Dynamics, Clarendon Press, Oxford, 1990.

[6] J. Fowler, H. Moon, and C.-J. Kim. (2002) "Enhancement of Mixing by Droplet-Based Microfluidics", in Digest Tech. Papaers IEEE MEMS 02 Conference, Las Vegas, Jan. 21-23, 2002, pp. 97-100.

[7] P. Paik, V. K. Pamula, and R. B. Fair, "Electrowettingbased droplet mixers for microfluidic systems", Lab on a Chip, vol. 4, pp. 253-259, 2003.

[8] J. G. Santiago, S. T. Wereley, C. D. Meinhart, D. J. Beebe, and R. J. Adrian, "A PIV system for microfluidics", Exp. in Fluids, vol. 25, pp. 316-319, 1998.

[9] C. D. Meinhart, S. T. Wereley, and J. G. Santiago "PIV measurements of a microchannel flow", Exp. in fluids, vol. 27, pp. 414-419, 1999.

[10] S. K. Fan, C. Hashi , and C.-J. Kim (2003) "Manipulation of multiple droplets on nxm grid by cross-reference EWOD driving scheme and pressure-contact packaging", in Digest Tech. Papers IEEE MEMS 03 Conference, Kyoto, Jan 19-23, 2003, pp. 694-697. 